\section{EUNUCHEN-STUDIE}

\section{Hoden verloren, Lebenszeit gewonnen}

\author{
Männer sterben im Mittel früher als \\ Frauen. Dieses Manko lässt sich offenbar \\ durch einen kleinen Eingriff beheben: \\ die Kastration.
}

- Aus Tierversuchen ist bekannt, dass eine Kastration und der damit verbundene Verlust an männlichen Sexualhormonen das Leben der betroffenen Tiere verglichen mit der Lebensspanne nicht kastrierter männlicher Artgenossen verlängert. Am Menschen ist das bisher wenig erforscht worden. Die Annahme, dass dies wenigstens zum Teil auf einen Mangel an Freiwilligen für einschlägige Studien zurückzuführen ist, dürfte nicht weit neben der Wahrheit liegen.

Koreanische Forscher haben deshalb einen historischen Kniff angewendet und sich in vergangenen Jahrhunderten umgesehen, wo die Kastration nicht unbedingt eine Sache der Freiwilligkeit gewesen war. Hierfür zogen sie die Daten der Yang-Se-Gye-Bo heran, eines zu Beginn des 19. Jahrhunderts niedergeschriebenen Stammbaums

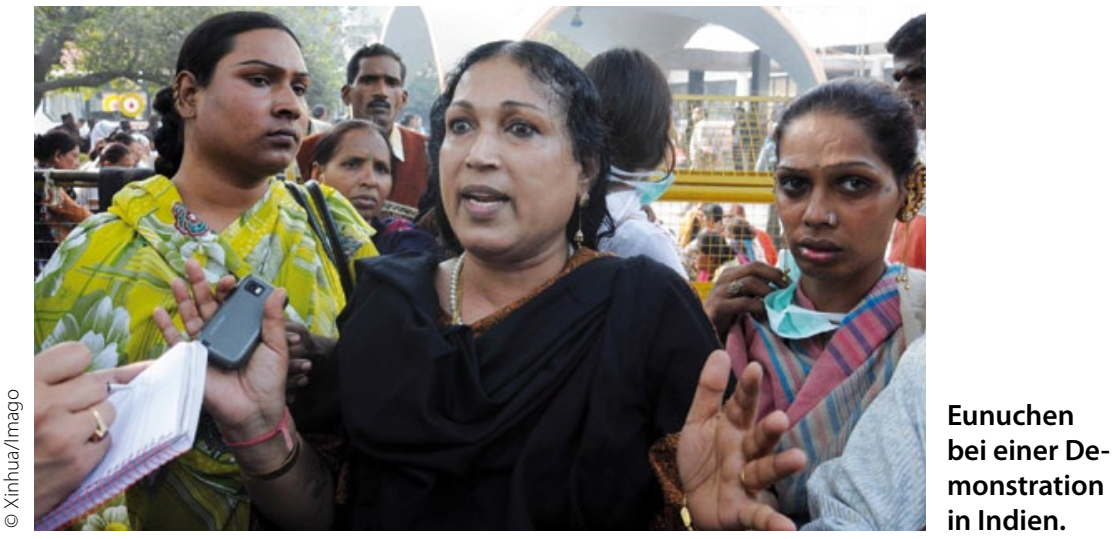

von Eunuchen über 13 Generationen. Stammbaum und Eunuchen klingt zunächst widersinnig, ist es aber nur halb. Denn in Korea war den Eunuchen die Heirat ebenso erlaubt wie das Adoptieren von Kindern - Mädchen oder kastrierte Knaben, versteht sich.

Aus der Genealogie ließen sich die Lebensspannen von 81 Eunuchen errechnen. Im Mittel wurden sie 70 Jahre alt, bei einem Bereich von 29 bis 109 Jahren. Damit verglichen wurden die Lebenszeiten von nicht kastrierten Männern aus drei Familien, die unter ähnlich privilegierten Umständen lebten wie die Eunuchen selbst. Das durchschnittlich erreichte Alter lag hier nur bei rund 56 Jahren, 14 Jahre weniger als bei den hodenlosen Zeitgenossen.

„Die Ergebnisse unserer Studie sprechen für eine Art Tauschgeschäft zwischen Reproduktion und Altern", schreiben die koreanischen Forscher. Hiernach würde die Reproduktionsfähigkeit auf Kosten somatischer Reparaturmechanismen aufrechterhalten. Kurz: Testosteron senkt die Lebenserwartung. Ob diese Erkenntnis praktische Folgen haben und die Zahl der Hodenverweigerer steigen wird, bleibt abzuwarten.

$\mathrm{RB}$ -

Min K-J et al. Current Biology 2012, 22: R1-2

\title{
Handy statt Hausarzt?
}

Endlich ist es da: das sehnsüchtig erwartete neue iPhone 5. Es kann wirklich (fast) alles, ja man kann sogar damit telefonieren. Doch für alle, die gehofft hatten, damit ließe sich die hausärztliche Versorgung verbessern, müssen enttäuscht sein. Gerade die Gesundheitspolitiker, die den Hausarzt als eine im Aussterben befindliches Gattung ansehen, hatten große Erwartungen an das neue Handy geknüpft und dieses auch mit dem Slogan „Handy statt Hausarzt" als modernes, flexibles, individuelles und zeitnahes ärztliches Versorgungskonzept i. S. eines individualisierten Telemonitorings propagiert. So glaubte man, Patienten mit einer zielgerichteten Sensibilisierung auf den kommenden

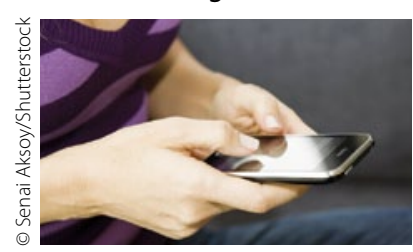

Bei Krankheit lieber zum Arzt!
Hausärztemangel vorbereiten und gleichzeitig Ängste abbauen zu können. Doch jetzt heißt es zurückrudern, April, April!!

Denn nichts von dem, was man von einer hausärztlichen Basisversorgung erwarten kann, konnte Apple seinem neuen
Handy beibringen. Keine digitale Spracherkennung, welche die Anamnese aufzeichnet und sofort eine Verdachtsdiagnose „ausspuckt". Keine kontinuierliche Blutdruck- und Pulsmessung, keine automatische Auskultation von Herz, Lunge oder Bauch, kein Schlafmonitoring, keine Sofortanalyse von Blut, Urin und Stuhl. Auch bei der Früherkennung psychischer Erkrankungen versagt das Handy, da es keine Möglichkeit bietet, den Gesichtsausdruck im Hinblick auf das Vorliegen einer affektiven Störung zu analysieren. Auch vermisst man die Option, über eine App Leistungen delegieren oder substituieren zu können. Gleiches gilt für das Service-Management wie das Ausstellen von Rezepten und Bescheinigungen. Kurzum, das biologische Wesen "Hausarzt" kann bis auf Weiteres durch das digitale Wesen „Handy“ nicht ersetzt werden. Und wenn man an die Kosten denkt, ist dies ja auch gut so; denn der billigste Handy-Vertrag mit Mondschein-Tarif ist immer noch teurer als die monatliche "Rund-um-Versorgung" durch den Hausarzt, gar nicht zu reden von den Anschaffungskosten!
Dr. med. Peter Stiefelhagen a 